\title{
INVESTIGATING SOCIAL DYNAMICS AND GLOBAL CONNECTIVITY: AN AGENT-BASED MODELING APPROACH
}

\author{
John A. Sokolowski \\ Catherine M. Banks \\ Virginia Modeling, Analysis and Simulation Center \\ Old Dominion University \\ 1030 University Blvd. \\ Suffolk, VA 23435, USA
}

\begin{abstract}
Social scientists have been exploring the factors that contribute to the assimilation and standardization of population as they affect a populace over a period of time. Research in this field has made the case for employing agent-based models to investigate this social phenomena. This research builds on the basic tenants of these previous approaches as a way to investigate the nature of global connectivity as it affects the standardization of a strong central culture of a specific region. The simulation runs conducted in this study make clear that the more cultural features that "characterize" agents, the less likely it is for their cultures to assimilate. The simulations also indicate that even when a culture has a $50 \%-60 \%$ chance of being attracted to the features of an outside culture, almost total cultural migration is possible. The paper presents an agent-based modeling approach to analyze dynamic (fluid) cultural change vis-à-vis global connectivity.
\end{abstract}

\section{INTRODUCTION}

Social scientists have been exploring the factors that contribute to the assimilation and standardization of population as these factors interact over a period of time on a population. It is interesting to note that this process can be interrupted and stopped short of complete homogeneity. Political Scientist Robert Axelrod developed an agent-based model to investigate the characteristics that cause cultural disparity to become less disparate. One of his conclusions was that communication is most effective when people share similar cultural features and the traits that define those features (Axelrod 1997). Basically, the spread of cultural features from one group to another is dependent on the number of features they already have in common. Further, this dependency is a function of the number of cultural features, the range of interactions, and the size of the geographic region. Several others have developed models of this social behavior (Carley 1991, 1995; Geertz 1973; Rogers 1983; Dignum and Dignum 2009). Axelrod's model of social influence differs from earlier proposed research in two ways: it explicitly accounts for the effect of one cultural feature on another; and it gives due consideration to the fact that individuals with similar features are more likely to influence each other than individuals with dissimilar features (Homans 1950).

Axelrod's model assumes that one can characterize culture by a series of attributes or features such as language, religion, ethnicity, etc. For each of these features there is a set of traits, which are values that a feature may take on. For example, religion may be one such feature that could have the following traits: Catholic, Protestant, Muslim, and Hindu. Further, Axelrod proposed that culture can be abstracted into a sequence of digits (features), each with a number representing possible trait values. For example, one such sequence could be 64823 indicating a culture composed of five features. The numbers represent an index to a particular trait for that feature. 


\section{Sokolowski and Banks}

The Axelrod model also included a geographic distribution of agents on a square grid with each grid square representing a homogeneous village characterized by this digit sequence. Each site was allowed to interact with its immediate neighboring cells to the north, south, east, and west of it. The chance of interaction by this cell with one of its neighbors is proportional to its cultural similarity as represented by the sequence of digits. If an interaction occurred, then the active cell would take on the trait of one of the features that was different in the culture of one of its randomly chosen neighbors. What emerged from these interactions were regions of shared culture. In many cases more than one stable region emerged. The number of emerging regions decreased with an increasing number of features while the number of emerging regions increased with an increasing number of traits per feature. Axelrod's model also showed that with increasing ranges of interaction came fewer stable regions, which implied that cultural convergence was easier when interactions occur at greater distances. Size of territory was also varied but produced no substantial difference on the number of stable regions. Therein is a constraint in the model: the Axelrod model encapsulates local interactions only-it does not address the possible effect of global interactions (connectivity) on the merging cultures.

To counter that constraint Gonzalez-Avella adapted Axelrod's model to include global as well as local coupling (Gonzalez-Avella et al. 2006). This model also incorporated the concept of global coupling which has been considered as representative of the influence of mass media across cultures (Shibanai, Yasuno, and Ishiguro 2001). In the Gonzalez-Avella model a vector field was added to the Axelrod model, which was treated as an additional neighbor to each geographic agent cell. The strength of the field was given by a constant parameter that measured the probability of interaction between a randomly chosen cell and the vector field.

The Gonzalez-Avilla model distinguished three types of fields. The first type represented an external field that was spatially uniform and constant over time. It was meant to represent a specific cultural state being imposed by a controlled mass media on all cells of the model. The second type, called a global field, was spatially uniform but allowed to vary over time. In the context of social dynamics this field contained the predominant trait in each cultural feature and it could represent a global media influence that has the potential of affecting every agent cell in a time-varying manner. The third type was a local field meant to represent local mass media conveying local cultural trends among immediate neighbors. In the context of Axelrod's model, the Gonzalez-Avilla experiment showed that the external and global mass media representations had similar effects on the social system. Namely, when the probability of interaction was sufficiently large, mass media actually contributed to cultural diversity. It was only efficient at producing cultural homogeneity in conditions of weak coupling. Local mass media effects were more effective in producing uniformity than the other two types.

Both Axelrod and Gonzalez-Avella et al. made a viable case for employing agent-based models to investigate this social phenomena. Hence, the work contained in this paper builds on the basic tenants of their approaches as a way to investigate the nature of global connectivity as it affects a strong central culture of a specific region. The method used in this paper is similar to the external field model (GonzalezAvella) described above. However, it is unique to that model approach and the original Axelrod approach in that it represents cultural traits by a measuring scale and not by specific enumerated sets. The measuring scale will serve to measure cultural disparity. This type of measurement methodology allows for fluidity to express the adoption (or adaptation) of traits as well as the reversal or recession of those traits. As such, the focus of this research is to introduce an evolutionary model that can represent the affects of global connectivity on cultural disparity. (As such, local influences will not be a component of this model.)

Also new to this study is the introduction and application of the concept standardization of population. The authors define standardization of population as a general population's gradual and evolutionary acceptance via adaptation or adoption of norms introduced from external influences be they social, political, cultural, religious. Standardization can take place by direct and indirect means. For example, a direct means could involve the use of hard power to initiate a revolution as a means of displacing the current government to make way for a different form of rule: i.e., remove the dictator to introduce and educate for 


\section{Sokolowski and Banks}

a democratic means of governance so that the general population comes to understand how and why this form of government could serve them more effectively. This direct means is premised on the hard power of the culture seeking to influence the population. A less pronounced direct means of standardization can come from within the culture itself as its political, social, and religious leaders seek to reconcile a disparate population: i.e., various tribal leaders endeavoring to cooperate for the greater good of the state or region. This can be done internally without external influences with the goal of ceasing hostilities and/or poor relations among groups that share various traits within a feature.

Indirect means of standardizing a population usually comes in the form of soft power, or the ability to obtain what one wants through co-option and attraction (Nye 2004). Often, the strength of soft power lies in the depth of desire a population ripe for influence has for the traits of another feature. For example, a once closed-society like Japan became ripe or desirous of certain traits in the feature, western culture. Twice in its modern history (1850s and 1980s) Japan conducted a quick integration of western (many American) traits. The ability to quickly absorb via direct adoption of some American standards proved the Japanese desire to look, think, and feel western. What was not adopted was adapted; accordingly, a walk through busy Tokyo entails a reflection of many things American. Thus, direct or indirect means of standardizing a population can include overt or covert activities, hard power or soft power.

Another indirect means of standardizing a population is found in the telecommunications capability that has fast-tracked the networking of societies at every level: local, national, regional, global. This is another form of soft power that contributes to the global connectivity the world has been experiencing during the past two decades. Just two decades ago the term globalization replaced the Cold War word internationalism in that borders became less meaningful as economies and communications saw the need for transparency. Today, globalization has entered the period of global connectedness because more than just goods cross borders-ideas and information seem to effortlessly make their way into the hands of "connected" citizens who have access to internet capability. A Global 2.0 world now exists and it is transforming (directly or indirectly) cultures, economies, and politics of billions of people (Amelio 2010). As with all soft data, integrating this concept / phenomena of global connectivity into a representation (model) of a real-world event is challenging. Still, it is necessary as a way to accurately measure the standardization of population.

The following discussion introduces the modeling approach and the results of a series of agent-based simulations to show the effect of global connectivity on cultural disparity (section 2). The next section (3) provides the results of a series of agent-based simulations, and the final section (4) presents conclusions and recommendations for further investigation of the effects of global connectivity.

\section{THE MODEL}

The basic structure of this model is similar to that of Axelrod's in that it has agents arrayed on a square grid. Each cell of the grid contains one agent that acts in an autonomous manner with other agents in the system. Each agent has a cultural identity represented by a set of $N$ abstract features that define its culture. There are two other special agents contained in this model. These agents represent differing cultures in that each feature of these agents has a consistent degree of separation between each of them. One can think of these two agents as deep-rooted and diametrically opposite cultural identities that do not change over time. Initially all agents on the grid have theirs features aligned to one of these differing cultures.

This model differs from the Axelrod model in that it does not have a set of traits that each feature can take on. Instead, the features have a Likert scale value that represents the degree of separation between the agent and a reference agent (one of the two special agents noted above). Sokolowski and Banks used a similar approach to characterize social and cultural differences affecting a country's susceptibility to insurgency (Sokolowski and Banks 2009). A Likert scale approach was chosen for this research because it facilitates a more thorough assessment of how one culture migrates to another given the influence of global connectivity on a target culture. This "influence" comes in the form of a culture significantly different than the target culture. The target culture may adopt, adapt or both aspects (traits) of the external, influencing culture. The target culture can also resist or regress from adopting or adapting aspects of the 


\section{Sokolowski and Banks}

influential culture due to interactions with its own culture acting as a push/pull in response to the influencing culture.

Thus, this model endeavors to characterize social influence and global connectivity in the following way:

1. Set the value for the degree of global connectivity, $B$. One can interpret this value as the probability that global connectivity has to attract the attention of an agent to an outside culture.

2. Set the feature values for each of the two special agents.

3. Randomly select an agent on the grid. Call this agent the active agent.

4. With probability $B$ the active agent interacts with the special agent whose culture is different than its own. This interaction consists of selecting a feature at random and incrementing its value by one such that it moves closer to the special agent's cultural value. This interaction represents a movement towards the culture being represented via global connectivity.

5. Calculate the cultural overlap between the active agent and the special agent representing its original culture. The overlap is the ratio of the number of common features between the active agent and its original culture agent to the total number of features.

6. With probability equal to the cultural overlap the active agent interacts with its original culture special agent. The interaction consists of selecting a feature at random that has a value different than the special agent and incrementing that value by one such that it moves closer to the special agent's cultural value. This interaction represents the active agent being influenced by how closely it is aligned to its native culture and moving back towards that culture.

7. Return to step 3 and repeat for the desired number of events.

To illustrate this procedure consider the following example. Suppose there are two cultures, $U$ and $I$, that are diametrically opposite to one another. The special agent representing Culture $U$ consists of the feature set $\left[\begin{array}{llll}5 & 5 & 5 & 5\end{array}\right]$. The special agent representing Culture $I$ has a feature set $\left[\begin{array}{lllll}1 & 1 & 1 & 1 & 1\end{array}\right]$. This indicates that there are five distinct features describing culture with a Likert scale of $1-5$ showing the separation of the features from Culture $U$ to culture $I$. Furthermore, Culture $I$ is the one that is being influenced by Culture $U$ via various global connection means. The agents on the grid have feature values initially set to that of Culture $I$. An active agent is chosen at random and interacts with special agent $U$ with probability $B$. The active agent's feature set may then look as follows: $\left[\begin{array}{lllll}1 & 2 & 1 & 1 & 1\end{array}\right]$. The cultural overlap with its native culture would then be eighty percent. The active agent then interacts with special agent $I$ with probability of $80 \%$. If this interaction occurs features are selected at random until one that is different from agent $I$ is found. In this case the resultant feature set would become [ $\left[\begin{array}{lllll}1 & 1 & 1 & 1 & 1\end{array}\right]$ for the active agent. As this process is repeated an emergent view of how global connectedness causes one culture to migrate towards another culture is created.

Before looking at simulation results for this model it is important to identify a measure that characterizes the migration of one culture towards another. Suppose one of the grid agents had the following feature vector: $\left[\begin{array}{lllll}3 & 3 & 3 & 3 & 3\end{array}\right]$. From a Likert scale interpretation this agent would be equidistant or neutral between the two cultures. If a subsequent interaction causes this agent's feature set to become $\left[\begin{array}{lllll}3 & 3 & 4 & 3 & 3\end{array}\right]$ then it would be closer to Culture $U$ than its original Culture $I$. That agent would then be considered as having migrated to the new culture. This measure of migration will be used to compute the number of agents that have moved to the new culture. Keep in mind that in subsequent events this agent could migrate back to its original culture as long as it is not completely aligned to the new culture, i.e. its feature set is not $\left[\begin{array}{lllll}5 & 5 & 5 & 5 & 5\end{array}\right]$.

\section{THE EFFECT OF GLOBAL CONNECTIVITY}

It is obvious from the above discussion that if global connectivity does not exist, migration will not occur. Thus, the research questions become clear: As the level of global connectivity increases, what will its im- 
pact be on the influenced (ripe) culture? Is there a threshold value where total migration occurs? Do the number of features affect the rate or level of migration?

To begin to answer these questions it is helpful to examine a single run of the simulation. The run begins with all agents having the same cultural feature values as shown in Figure 1a. As agents begin to interact with the special agents their feature values change. The shades of grey for each agent cell in Figure 1 indicate the level of migration that an agent has towards the other culture. The lighter the shade of grey, the higher the migration level. In the case of this example the higher event counts tend to indicate that migration is increasing over time. However, one cannot tell at this point if any kind of equilibrium condition has been reached. To determine this a systematic investigation of how the various model parameters affect the outcome of the simulation must be done.

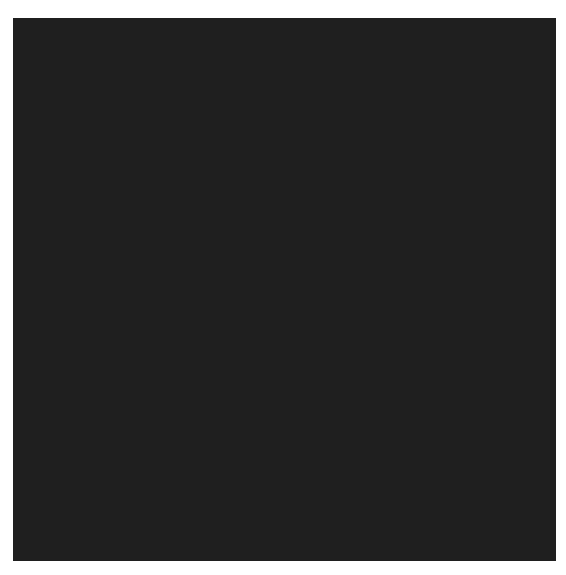

(a) At start

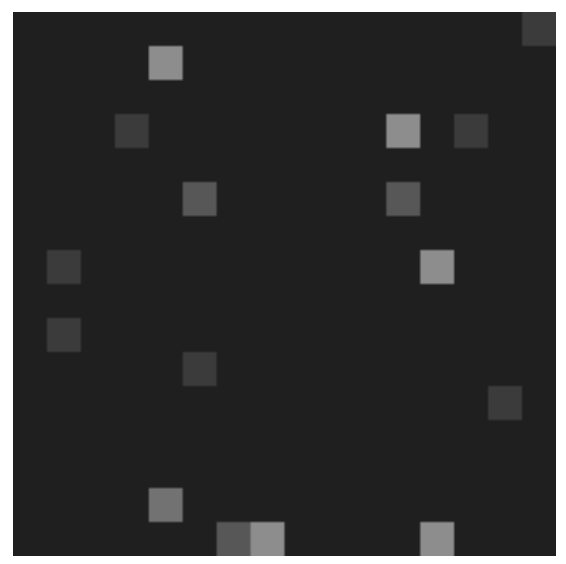

(c) After 40,000 events

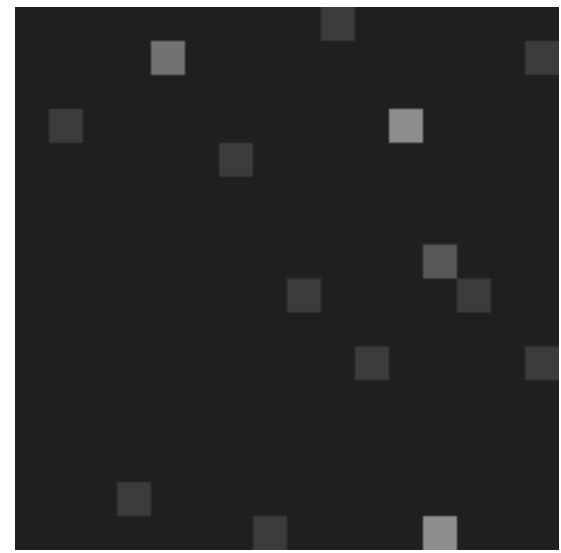

(b) After 20,000 events

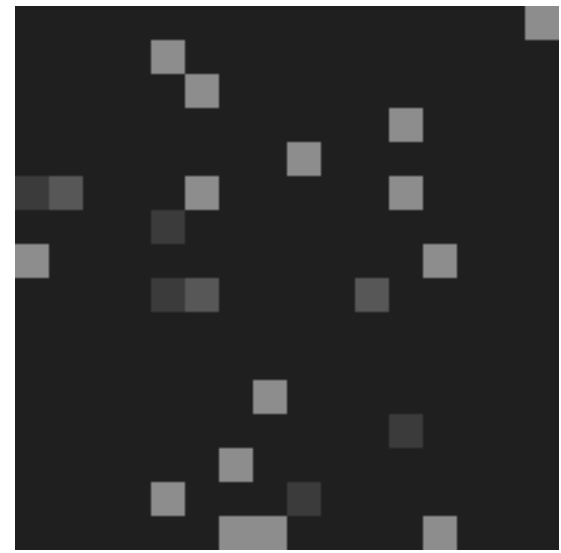

(d) After 80,000 events

Figure 1: Agents in various stages of migration

Of primary importance in this research is how the level of global connectedness affects cultural change. To investigate this question a simulation was set up using the model described above. A $16 \times 16$ grid was selected as the test area thus providing 256 cells each containing one agent. The number of features describing each agent's culture was set at 5. Two special agents were instantiated. Agent $U$ represented the culture from which global information was being disseminated and was assigned the following set of feature values: $\left[\begin{array}{lllll}5 & 5 & 5 & 5 & 5\end{array}\right]$. Agent $I$ represented the culture of which each agent on the grid 
originally belonged. This agent and all grid agents were assigned feature values $\left[\begin{array}{lllll}1 & 1 & 1 & 1 & 1\end{array}\right]$. The degree of global connectedness, $B$, was varied from 0.1 to 0.6 and ten runs were conducted for each value of $B$. The average fraction of migration (average number of agents that migrated/total number of agents) was then computed for each of the tested connectedness values. Table 1 provides the results for each global connectedness value. Figure 2 is a plot of these results.

Table 1: Global Connectedness Results

\begin{tabular}{|l|l|}
\hline Degree of Global Connectedness & Fraction of Migration \\
\hline 0.1 & 0 \\
\hline 0.2 & 0.0066 \\
\hline 0.25 & 0.0266 \\
\hline 0.3 & 0.0758 \\
\hline 0.4 & 0.4031 \\
\hline 0.5 & 0.8484 \\
\hline 0.6 & 0.9926 \\
\hline
\end{tabular}

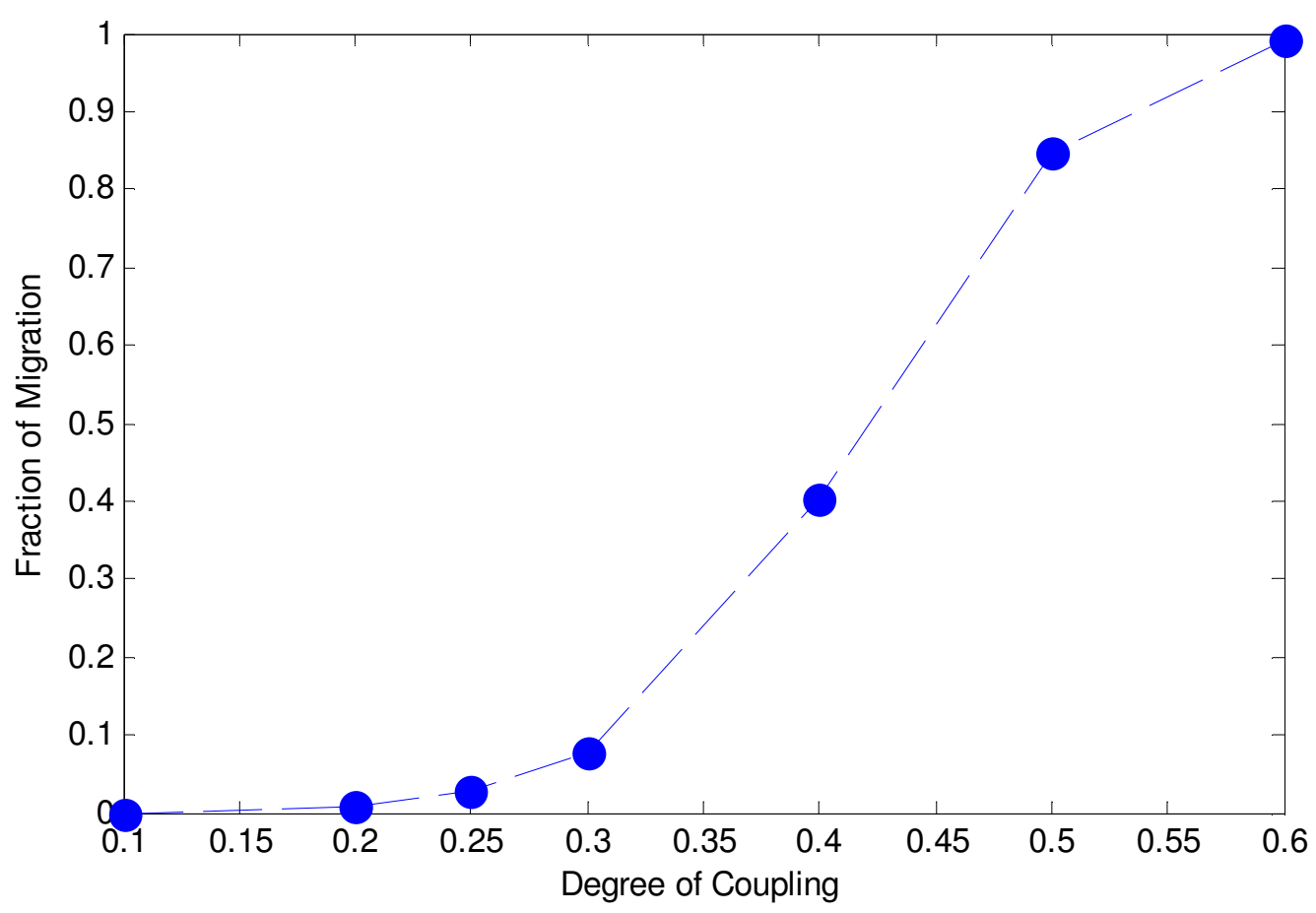

Figure 2: Plot of Global Connectedness Results

Notable is the fact that significant migration does not begin to occur until the degree of coupling surpasses $30 \%$. At that point migration increases rapidly with almost complete migration taking place near $60 \%$ coupling. A somewhat surprising result is that this level of migration is reached with just above a $50 \%$ interaction probability even in the presence of a stable culture counteracting the affects of the counter culture.

The second question investigated concerns how the number of features affect the migration fraction for a given degree of coupling. The simulation was set up in a similar manner to that above, i.e. a $16 \times 16$ 
grid with the same initial conditions for the special agents. A 0.4 connectedness factor was chosen since that factor provided a reasonable chance of discernment given the relatively large number of migrations that take place at that level. In addition to the initial run of the simulation using 5 features the simulation ran with 6,7,8, and 10 features selected again with 10 runs each. Results of these simulations are provided in Table 2.

Table 2: Migration Fraction vs. Number of Features

\begin{tabular}{|l|l|}
\hline Number of Features & Fraction of Migration \\
\hline 5 & 0.4031 \\
\hline 6 & 0.1449 \\
\hline 7 & 0.0422 \\
\hline 8 & 0.0141 \\
\hline 10 & 0.0027 \\
\hline
\end{tabular}

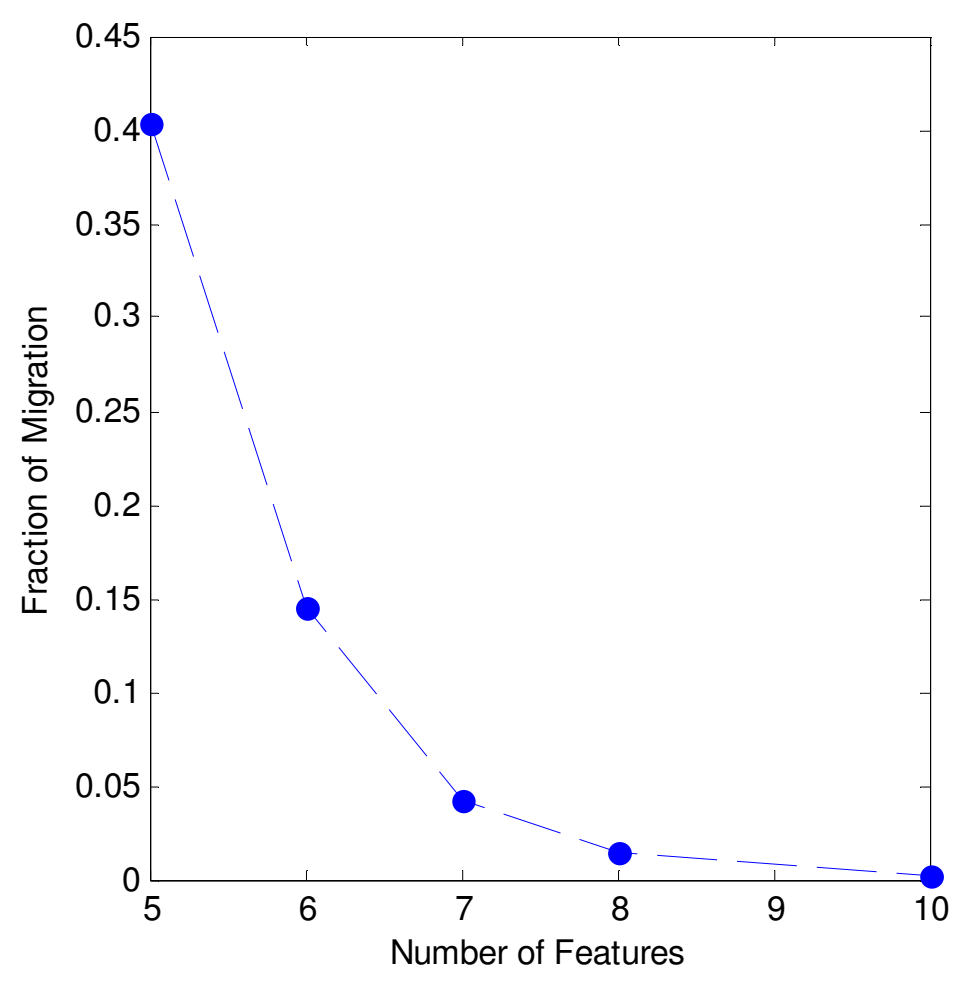

Figure 3: Impact of Features on Migration

One can see from these results that as the number of features used to describe a culture increases less migration takes place for a given percentage of connectivity. Intuitively this result was expected because a greater number of feature changes must occur over a given period of time to achieve the same level of migration.

\section{CONCLUSIONS}

From the results of these runs it is obvious that when more cultural features that characterize agents exist, the less likely it is for their cultures to assimilate. This result is contrary to that of the GonzalezAvella model. Their results indicated that when the probability of mass media interaction is large enough, 


\section{Sokolowski and Banks}

cultural diversity increases. While the two models are not directly comparable because of how each model differs in its measurement of diversity, they are close enough in concept that further investigation is warranted to explain the conflicting results.

Of equal importance is the response of migration to the degree of connectedness for a given number of features. When the probability of attracting the attention of an agent to an outside culture is only in the range of 0.50 to 0.60 , there is near total migration towards that opposite culture even in the presence of a strong local cultural pull. This result has significant implications to the study of cultural migration in this ever increasingly connected world and the desire to understand how these changes will affect the global community.

As such, future work in this area should include adding local agent interaction to our model to investigate its impact on cultural migration and validation of the model by comparison of its results to that of a real world case study.

\section{REFERENCES}

Amelio, W. 2010. Interconnected We Prosper. New York Times.

Axelrod, R. 1997. The Dissemination of Culture: A Model with Local Convergence and Global Polarization. Journal of Conflict Resolution 41 (2):203-226.

Carley, K. 1991. A Theory of Group Stability. American Sociological Review 56:331-354.

1995. Communication Technologies and their Effect on Cultural Homogeneity, Consensus, and the Diffusion of New Ideas. Sociological Perspectives 38 (4):547-571.

Dignum, F., and V. Dignum. 2009. Emergence and Enforcement of Social Behavior. In Eighteenth World IMACS/MODSIM Congress. Cairns, Australia.

Geertz, C. 1973. The Interpretation of Cultures. New York: Basic Books.

Gonzalez-Avella, J. C., V. M. Egufluz, M. G. Cosenza, K. Klemm, J. L. Herrera, and M. San Miguel. 2006. Local Versus Global Interactions in Nonequilibrium Transitions: A Model of Social Dynamics. Physical Review E 73 (4):046119-1 - 046119-7.

Homans, G. C. 1950. The Human Group. New York: Harcourt Brace.

Nye, J. 2004. Soft Power: The Means to Success in World Politics. Boston: Harvard University Press.

Rogers, E. M. 1983. Diffusion of Innovations. 3d ed. New York: Free Press.

Shibanai, Y., S. Yasuno, and I. Ishiguro. 2001. Effects of Global Information Feedback on Diversity: Extensions to Axelrod's Adaptive Culture Model. Journal of Conflict Resolution 45:80-96.

Sokolowski, J. A., and C. M. Banks. 2009. A Methodology to Explore the Impact of Policy Changes on Insurgencies. International Journal of System of Systems Engineering 1 (3):314-328.

\section{AUTHOR BIOGRAPHIES}

JOHN A. SOKOLOWSKI is the Executive Director of Old Dominion University's Virginia Modeling, Analysis \& Simulation Center (VMASC). He holds a Bachelor of Science degree in Computer Science from Purdue University, a Master of Engineering Management from Old Dominion University (ODU), and a Ph.D. in Engineering with a concentration in the Modeling and Simulation from ODU. His research interests include human behavior modeling, multi-agent system simulation, and simulation techniques for representing social systems.

CATHERINE BANKS is a Research Assistant Professor at Old Dominion University's Virginia Modeling, Analysis, and Simulation Center. She is also an adjunct associate professor of Political Science and adjunct assistant professor of Modeling and Simulation at Old Dominion University. Dr. Banks received her Ph.D. in International Studies. Her current research focuses on modeling states and their varied histories of revolution and insurgency, political economy and state volatility, homeland security (emergency preparedness and management), and population dynamics. 